\title{
Insuficiencia cardiaca aguda en el anciano: características clínicas y mortalidad según la fracción de eyección ventricular izquierda
}

\author{
Jonathan Franco ${ }^{a, *}$, Francesc Formiga ${ }^{\mathrm{b}}$, David Chivite ${ }^{\mathrm{b}}$, Xavier Corbella $^{\mathrm{b}, \mathrm{c}}$, \\ Jordi Robert ${ }^{\mathrm{a}}$, Antonio Vidaller ${ }^{\mathrm{a}}$ y Ángel Charte ${ }^{\mathrm{a}}$
}

\author{
a Servicio de Medicina Interna. Hospital Quiron Dexeus, Barcelona, España \\ b Servicio de Medicina Interna. Hospital Universitari de Bellvitge. L'Hospitalet de Llobregat, Barcelona, España \\ c Facultad de Medicina y Ciencias de la Salud, Universitat Internacional de Catalunya, Barcelona, España
}

Recibido el 6 de agosto de 2016; aceptado el 24 de octubre de 2016

Disponible en Internet el 5 de enero de 2017

\section{PALABRAS CLAVE \\ Insuficiencia \\ cardiaca; \\ Anciano; \\ Fracción de eyección \\ ventricular izquierda}

\begin{abstract}
Resumen
Objetivos: Describir y analizar las características clínicas de la insuficiencia cardiaca en el anciano, así mismo, la influencia en la mortalidad según su fracción de eyección ventricular izquierda.

Método: Estudio ecológico multigrupo descriptivo en 115 pacientes hospitalizados por la insuficiencia cardiaca aguda con un período de seguimiento de un año. Según la fracción de eyección ventricular izquierda los pacientes se dividieron en dos grupos (fracción de eyección ventricular izquierda preservada y no preservada, si presentaban $\geq 50 \% 0<50 \%$, respectivamente). Se recogió la tasa de mortalidad al año del alta hospitalaria y se realizó un análisis de Cox de mortalidad.

Resultados: La edad media fue de 83,9 años y el 40\% fueron hombres. En 76 (66\%) pacientes la fracción de eyección ventricular izquierda era preservada. Al año, 35 pacientes $(30,4 \%)$ fallecieron. En el grupo de la fracción de eyección ventricular izquierda preservada predominaron más las mujeres y presentaron más comorbilidades como: la hipertensión arterial, la diabetes mellitus, la fibrilación auricular y la enfermedad pulmonar obstructiva crónica y por otro lado presentaron menos cardiopatía isquémica. Aunque la mortalidad era mayor en el grupo de la fracción de eyección ventricular izquierda no preservada, no se alcanzaba significación estadística $(38,5 \%$ vs. $26,3 \% ; p=0,180)$.
\end{abstract}

\footnotetext{
* Autor para correspondencia.

Correo electrónico: Jhonatanfrancov@gmail.com (J. Franco).
} 
Conclusiones: La mortalidad al año de los pacientes ancianos con insuficiencia cardiaca es alta, así mismo tienen una mayor proporción de la fracción de eyección ventricular izquierda preservada, que a la vez asocia un mayor número de comorbilidades y una tendencia a mayor supervivencia al año con respecto a la fracción de eyección ventricular izquierda no preservada. (c) 2016 Sociedad Colombiana de Cardiología y Cirugía Cardiovascular. Publicado por Elsevier España, S.L.U. Este es un artículo Open Access bajo la licencia CC BY-NC-ND (http:// creativecommons.org/licenses/by-nc-nd/4.0/).

\section{KEYWORDS}

Heart failure;

Elderly;

Left ventricular

ejection fraction

\section{Introducción}

En la última década la prevalencia de la insuficiencia cardiaca, se ha desplazado paulatinamente hacia el grupo de pacientes con más edad, con una proporción importante en los ancianos que alcanzan cifras entre el $22 \%$ en $>80$ años $^{1,2}$. En la insuficiencia cardiaca aguda, después de un episodio de descompensación, suele existir un deterioro funcional con una alta tasa de morbimortalidad hospitalaria ${ }^{3}$. Los reingresos pueden alcanzar un $16-50 \%$ y la mortalidad un $30-40 \%$ durante el primer año ${ }^{4}$, por ello cada vez se buscan con más frecuencia marcadores pronósticos ${ }^{5}$.

La aparición de nuevas dianas terapéuticas en la insuficiencia cardiaca, han contribuido a mejorar el pronóstico y la supervivencia en los pacientes, llevando esto a encontrarnos a pacientes de edad más avanzada. La presencia de la insuficiencia cardiaca en personas mayores de 75 años tiene una reducción del $50 \%$ de su expectativa de vida ${ }^{6}$. Tras un ingreso por insuficiencia cardiaca aguda la mortalidad al año en octogenarios alcanza entre un 24 a $50 \%{ }^{7}$.

El diagnóstico de la insuficiencia cardiaca en los ancianos es un reto clínico, teniendo en cuenta las manifestaciones clínicas "atípicas", que sumado a sus altas comorbilidades o a los cambios fisiológicos debidos a la senescencia, pueden disminuir la sensibilidad y especificidad de los síntomas clásicos de la insuficiencia cardiaca ${ }^{8}$.

Existen pocos datos sobre los perfiles clínicos, pronósticos y el impacto de intervenciones terapéuticas habituales en esta población ${ }^{9}$. Nuestro trabajo busca evaluar en un grupo de octogenarios, las características clínicas y la mortalidad de esta epidemia que es la insuficiencia cardiaca en una población excluida en la mayoría de las series bibliográficas.

\section{Métodos}

\section{Muestra del estudio}

Los pacientes incluidos en el estudio pertenecían al registro del grupo de trabajo de la insuficiencia cardiaca y la fibrilación auricular del Hospital Bellvitge, Barcelona, España. Se trata de un registro prospectivo, que incluye los pacientes consecutivos ingresados por la insuficiencia cardiaca en el servicio de medicina interna ${ }^{10}$. El comité de ética de investigación clínica del hospital, aprobó los protocolos para la 
recolección de datos, y todos los pacientes dieron su consentimiento informado para ser incluidos.

El registro incluye a los pacientes de edad igual o superior a 50 años ingresados por la insuficiencia cardiaca aguda, según los criterios diagnósticos de la Sociedad Europea de Cardiología ${ }^{11}$.

Los pacientes que fallecieron durante el ingreso hospitalario fueron excluidos. Para este estudio se evaluaron los pacientes con edad $\geq 80$ años y que disponían de la ecocardiografía $2 \mathrm{D}$.

El seguimiento al alta hospitalaria consistió en dos visitas de control a los 3 y 12 meses. La mortalidad durante el seguimiento, fue considerada como objetivo principal. Se contactó con los pacientes (o sus familiares) cuando éstos no se presentaron a las visitas de control, para poder determinar las causas y su situación clínica. Los ingresos posteriores se codificaron como reingresos.

Se respetó la confidencialidad, ya que no se almacenaron datos personales, a excepción de la fecha de nacimiento e iniciales del nombre para evitar duplicación de datos.

El registro incluía: datos sociodemográficos, antecedentes patológicos, comorbilidades (Índice de Charlson), estado funcional basal para actividades básicas de la vida diaria (Índice de Barthel), datos clínicos de admisión (presión arterial, frecuencia cardiaca, peso y altura, índice de masa corporal, características de la descompensación, factores desencadenantes), complicaciones durante el ingreso y la prescripción de medicamentos. Con respecto a la severidad clínica de la insuficiencia cardiaca, se utilizó la clase funcional mediante la escala de la New York Heart Association, la evaluación de la fracción de eyección del ventrículo izquierdo mediante la ecocardiografía $2-D$, el índice cardiotorácico mediante la radiografía del tórax y las alteraciones del electrocardiograma. Las determinaciones analíticas incluían: hemograma, función renal, lípidos, glucosa, ácido úrico y troponina. El filtrado glomerular se estimó con la ecuación MDRD y la enfermedad renal crónica, se consideró cuando la tasa de filtrado glomerular estimado era inferior a $60 \mathrm{ml} / \mathrm{min} / 1,73 \mathrm{~m}^{2}$.

\section{Análisis estadístico}

Se trata de un estudio ecológico multigrupo descriptivo. Se utilizó la media y la desviación estándar para las variables cuantitativas y el porcentaje para las variables categóricas. Se compararon las características y la mortalidad al año de los pacientes según dos grupos: la fracción de eyección ventricular izquierda preservada ( $\geq 50 \%$ ) o no preservada (< $50 \%$ ). La comparación de las características de ambos grupos (fracción de eyección ventricular izquierda $\geq 50 \%$ o $<50 \%$ ) se realizó mediante las pruebas de $\chi 2$ y t de Student. Se utilizó un análisis de regresión logística y un análisis multivariante de riesgos proporcionales de regresión de Cox para evaluar la mortalidad intrahospitalaria y al año de seguimiento. El nivel de significación estadística se estableció en $\mathrm{p}<0,05$.

Para el análisis de los datos se utilizó el paquete estadístico Statistical Package for Social Sciences program (versión 21.0, Statistical Package for Social Sciences Inc. Chicago. IL, USA).

\section{Resultados}

Se incluyeron en total 115 pacientes de forma consecutiva: 69 mujeres $(60 \%)$ y 46 varones (40\%). La edad media fue de 83,9 años. En 76 pacientes (66\%) la fracción de eyección ventricular izquierda era preservada. La tabla 1 muestra las diferencias entre ambos grupos. En el grupo de la fracción de eyección ventricular izquierda preservada existía un predominio mayor de mujeres, así como de presentar en mayor proporción comorbilidades asociadas como: la hipertensión arterial, la diabetes mellitus, la fibrilación auricular y la enfermedad pulmonar obstructiva crónica, aunque por el contrario, presentaron menos cardiopatía isquémica. En cuanto al tratamiento, se observó en ambos grupos una alta prevalencia del uso de: diuréticos de asa, inhibidores de la enzima convertidora de angiotensina/bloqueadores de los receptores de angiotensina $॥$, mientras que poco uso de betabloqueadores y antialdosterónicos. La mortalidad total al año fue de 35 pacientes (30,4\%). La mortalidad tuvo una clara tendencia a ser mayor en el grupo con la fracción de eyección ventricular izquierda no preservada, pero sin alcanzar la significación estadística (tabla 1).

\section{Discusión}

En la actualidad, según varios estudios, la prevalencia de la insuficiencia cardiaca en el octogenario oscila entre un $22-40 \%$, convirtiéndose en la llamada "epidemia geriátrica'. No existen datos epidemiológicos que diferencien con certeza si en esta población geriátrica predomina la insuficiencia cardiaca con la fracción de eyección ventricular izquierda preservada versus con la fracción de eyección ventricular izquierda no preservada. En este estudio, existe una mayor proporción de pacientes $\geq 80$ años de edad con la fracción de eyección ventricular izquierda preservada, con el fenotipo clásico descrito en la literatura: predominio de género femenino y con un mayor porcentaje de comorbilidad. Con respecto a la etiología de la insuficiencia cardiaca en los pacientes con la fracción de eyección ventricular izquierda preservada, ésta presentaba mayor tendencia a ser de causa hipertensiva y/o valvular, mientras que en la fracción de eyección ventricular izquierda no preservada dominaba la causa isquémica. Este último dato puede estar infravalorado por requerir para el diagnóstico pruebas invasivas, que en ocasiones pueden estar contraindicadas por la presencia de comorbilidades y el estado funcional de estos enfermos.

Algunos estudios han demostrado que en pacientes $\geq 80$ años de edad, con insuficiencia cardiaca aguda existe una asociación de 5 o más enfermedades crónicas a la vez ${ }^{12}$. Dado que la comorbilidad en los pacientes octogenarios tiene una alta prevalencia, y que ésta aumenta en paralelo con la edad, parece razonable incorporar en la rutina habitual una valoración global de la pluripatología en el paciente anciano con insuficiencia cardiaca. En cuanto al tratamiento farmacológico, las guías no establecen diferencias en función de la edad del paciente, pues no hay muchos datos sobre la eficacia del tratamiento farmacológico en este grupo de pacientes ancianos. En este estudio, observamos poco uso de los betabloqueadores y los antialdosterónicos, probablemente en relación con la existencia de factores que limitan 
Tabla 1 Características diferenciales de los pacientes $\geq 80$ años con la insuficiencia cardiaca según la fracción de eyección del ventrículo izquierdo

\begin{tabular}{|c|c|c|c|c|}
\hline & Total $(n=115)$ & FEVI $\geq 50(n=76)$ & FEVI < $50(n=39)$ & $\mathrm{p}$ \\
\hline Edad, años & $83,9(3)$ & $83,7(2,9)$ & $83,9(3,3)$ & 0,808 \\
\hline Sexo femenino & $69(60 \%)$ & $49(64,5 \%)$ & $20(51,3 \%)$ & 0,172 \\
\hline MNA & $10,35(2,5)$ & $10,5(2,5)$ & $10(2,4)$ & 0,366 \\
\hline Etiología isquémica & $48(41,7 \%)$ & $24(31,6 \%)$ & $24(61 \%)$ & 0,002 \\
\hline Diabetes mellitus & $42(36,5 \%)$ & $28(36,8 \%)$ & $14(35,9 \%)$ & 0,921 \\
\hline Hipertensión arterial & $78(67,8 \%)$ & $27(69,2 \%)$ & $51(67,1 \%)$ & 0,817 \\
\hline EPOC & $30(26,1 \%)$ & $23(30,3 \%)$ & $7(17,9 \%)$ & 0,155 \\
\hline Anemia & $32(27,8 \%)$ & 19 (25\%) & $13(33,3 \%)$ & 0,345 \\
\hline Fibrilación auricular & $46(40 \%)$ & $34(44,7 \%)$ & $12(30,8 \%)$ & 0,008 \\
\hline IC de debut & $24(20,9 \%)$ & 19 (25\%) & $5(12,8 \%)$ & 0,128 \\
\hline Índice Charlson & $2,5(1,2)$ & $2,4(1,2)$ & $2,7(1,3)$ & 0,146 \\
\hline PAS, mm Hg & $146,1(33)$ & $149,4(28,9)$ & $149,3(40,1)$ & 0,455 \\
\hline FC, Lpm & $92(36,1)$ & $90,9(40,1)$ & $94,1(27)$ & 0,648 \\
\hline Hematocrito & $35,9(6,7)$ & $36(6,5)$ & $35,8(7,2)$ & 0,849 \\
\hline Creatinina, $\mathrm{mg} / \mathrm{dL}$ & $134,4(64,7)$ & $136,3(70,4)$ & $130,5(52,7)$ & 0,648 \\
\hline Sodio, meq/L & $139,3(5)$ & $139,8(4,2)$ & $138,5(6,3)$ & 0,202 \\
\hline Potasio, meq/L & $4,4(0,6)$ & $4,4(0,7)$ & $4,3(0,5)$ & 0,668 \\
\hline Urato, mg/dL & $9,41(3,6)$ & $9,7(3,8)$ & $8,8(3,1)$ & 0,238 \\
\hline Índice Barthel & $84,8(19,4)$ & $85,2(19,2)$ & $84,2(19,2)$ & 0,802 \\
\hline Diuréticos de Asa & $96(83,5)$ & $61(80,3)$ & $35(89,7)$ & 0,195 \\
\hline IECA/ARA ॥ & $88(76,5)$ & $53(69,7)$ & $35(89,7)$ & 0,017 \\
\hline Betabloqueadores & $22(31)$ & $13(28,9 \%)$ & $9(34,6 \%)$ & 0,615 \\
\hline Antialdosterónicos & $13(11,3)$ & $10(13,2 \%)$ & $3(7,7 \%)$ & 0,381 \\
\hline Mortalidad al año & $35(30,4 \%)$ & $20(26,3 \%)$ & $15(38,5 \%)$ & 0,180 \\
\hline
\end{tabular}

EPOC: enfermedad pulmonar obstructiva crónica; FEVI: fracción de eyección del ventrículo izquierdo; IC: insuficiencia cardiaca; FC: frecuencia cardiaca; IECA: inhibidores de la enzima convertidora de angiotensina; ARA ॥: antagonistas de receptores de angiotensina ॥. Los valores expresan n (\%) o media-desviación estándar.

la eficacia de este tipo de tratamiento farmacológico en los pacientes mayores, ya sea por la alteración de su farmacocinética y farmacodinamia en la edad avanzada, o la existencia de pluripatología, polifarmacia u otros factores sociales y funcionales que limitan la prescripción de algunos de estos medicamentos.

A pesar de estos avances en el tratamiento, la población anciana $\geq 80$ años con insuficiencia cardiaca sigue presentando una alta mortalidad, que alcanza el $30,4 \%$ en el total de nuestra cohorte tras el primer ingreso por insuficiencia cardiaca aguda, una cifra similar a otros estudios que presentan cifras de entre el $24-50 \%^{7}$. Apreciamos a su vez, una clara tendencia a presentar una mayor mortalidad en pacientes $\geq 80$ años de edad con insuficiencia cardiaca y la fracción de eyección ventricular izquierda no preservada (38,5\% vs. $23,6 \%$; p 0,180), aunque sin alcanzar la significación estadística. Estos datos obligan a abrir las puertas a futuros estudios y ensayos clínicos específicos para esta población anciana, que queda habitualmente excluida en la gran mayoría de grandes series, y los datos en las guías clínicas son escasos ${ }^{13,14}$.

En el momento de evaluar los resultados hay que tener en cuenta como limitaciones el hecho de que se trata de un estudio unicentro, lo que dificulta la generabilidad de los resultados y el hecho de que no se han evaluado los pacientes con la fracción de eyección ventricular izquierda intermedia de manera separada.

\section{Conclusión}

Nuestros resultados confirman la hipótesis de que los pacientes ancianos $\geq 80$ años con insuficiencia cardiaca presentan en mayor proporción una fracción de eyección ventricular izquierda preservada, asociada en un importante número de casos a diversas comorbilidades concomitantes, y a una tasa de mortalidad global al año de seguimiento de alrededor del 30\%. A la luz de todo ello, y para mejorar el abordaje global de estos pacientes, consideramos muy necesaria la incorporación definitiva de una valoración geriátrica multidisciplinar en las guías de práctica clínica para el manejo clínico de la insuficiencia cardiaca en la población anciana.

\section{Responsabilidades éticas}

Protección de personas y animales. Los autores declaran que para esta investigación no se han realizado experimentos en seres humanos ni en animales.

Confidencialidad de los datos. Los autores declaran que han seguido los protocolos de su centro de trabajo sobre la publicación de datos de pacientes. 
Derecho a la privacidad y consentimiento informado. Los autores declaran que en este artículo no aparecen datos de pacientes.

\section{Conflicto de intereses}

Los autores declaran no tener ningún conflicto de intereses.

\section{Bibliografia}

1. Wong CY, Chaudhry SI, Desai MM, Krumholz HM. Trends in comorbidity, disability, and polypharmacy in heart failure. Am J Med. 2011;124:136-43.

2. Chivite D, Formiga F. Pujol R. La insuficiencia cardiaca en el paciente anciano. Rev Clin Esp. 2011;211:26-35.

3. Setoguchi S, Stevenson LW. Hospitalizations in patients with heart failure: who and why. J Am Coll Cardiol. 2009;54:1703-5.

4. McMurray JJ, Pfeffer MA. Heart failure. Lancet. 2005;365:1877-89.

5. Jungbauer C, Riedlinger J, Block D, Stadler S, Birner C, Buesing $M$, et al. Panel of emerging cardiac biomarkers contributes for prognosis rather than diagnosis in chronic heart failure. Biomark Med. 2014;8:777-89.

6. Mahjoub H, Rusinaru D, Souliere V, Durier C, Peltier M, Tribouilloy $\mathrm{C}$. Long-term survival in patients older than 80 years hospitalised for heart failure. A 5-year prospective study. Eur J Heart Fail. 2008;10:78-84.
7. Shah RU, Tsai V, Klein L, Heidenreich PA. Characteristics and outcomes of very elderly patients after first hospitalization for heart failure. Circ Heart Fail. 2011;4:301-7.

8. Oudejans I, Mosterd A, Bloemen JA, Valk MJ, van Velzen E, Wielders JP, et al. Clinical evaluation of geriatric outpatients with suspected heart failure: value of symptoms, signs, and additional tests. Eur J Heart Fail. 2011;13:518-27.

9. Chivite D, Franco J, Formiga F. Chronic heart failure in the elderly patient. Rev Esp Geriatr Gerontol. 2015;50:237-46.

10. Franco J, Formiga F, Chivite D, Cerda P, Corbella X. Prognostic Role of Hyperuricemia in Acute Heart Failure. Rev Esp Cardiol. 2016;69:699-700.

11. McMurray JJ, Adamopoulos S, Anker SD, Auricchio A, Böhm M, Dickstein $\mathrm{K}$, et al. ESC guidelines for the diagnosis and treatment of acute and chronic heart failure 2012: The Task Force for the Diagnosis and Treatment of Acute and Chronic Heart Failure 2012 of the European Society of Cardiology. Developed in collaboration with the Heart Failure Association (HFA) of the ESC. Eur J Heart Fail. 2012;14:803-69.

12. Lang CC, Mancini DM. Non-cardiac comorbidities in chronic heart failure. Heart. 2007;93:665-71.

13. Trullàs JC, Formiga F, Pérez-Calvo JI, Manzano L. En representación del Grupo de Trabajo de Insuficiencia Cardiaca y Fibrilación auricular de la Sociedad Española de Medicina Interna. What do experts think about heart failure guidelines? Rev Clin Esp. 2016 May 18, http://dx.doi.org/10.1016/j.rce.2016.04.07.

14. Formiga F, Aramburu-Bodas O, Pérez-Calvo Jl. Heart failure in elderly patients: it is time to add geriatric assessment. Eur J Heart Fail. 2013;15:1075. 